\title{
Efficacy of Dinoprostone Vaginal Pessary for Cervical Ripening and Induction of Labour
}

\author{
Mamatha C. ${ }^{1}$, Sarmishta M. ${ }^{2}$, Dhanalakshmi M.G. ${ }^{3}$ \\ 1, 2, 3 Department of Obstetrics and Gynaecology, Sri Ramachandra \\ Medical University, Chennai, Tamilnadu, India.
}

\section{ABSTRACT}

\section{BACKGROUND}

To evaluate the efficacy of dinoprostone vaginal pessary for cervical ripening and labour induction in term pregnant women.

\section{METHODS}

This was a prospective observational study conducted at Sri Ramachandra Institute of Higher Education and Research from September 2016 to September 2018. The study included pregnant women with singleton pregnancy and gestational age (GA) between 37 weeks and 41 weeks of gestation. The estimated sample size was 100 . All 100 women were induced with dinoprostone vaginal pessary.

\section{RESULTS}

The average induction to active phase interval was 10 hours 58 minutes. About 60.3 $\%$ of primigravida had an active phase time interval of 12 hours 03 minutes; and 95.2 $\%$ of primigravida delivered $<24$ hours with a mean induction to delivery time interval of 17 hours 36 minutes. $4 \%$ of primigravida had failed induction. About 84.8 $\%$ of multigravida delivered with mean induction to active phase time interval of 08 hours 57 minutes and $100 \%$ of multigravida delivered $<24$ hours with a mean induction to delivery time interval of 12 hours 19 minutes. Out of 100 patients, $80 \%$ of patients delivered by spontaneous vaginal delivery with episiotomy, $5 \%$ of patients delivered by assisted vaginal delivery with episiotomy and $15 \%$ of patients delivered by Caesarean section. Almost $97 \%$ of multiparous women delivered vaginally. No adverse maternal and neonatal outcome was noted in the present study.

\section{CONCLUSIONS}

Dinoprostone vaginal pessary is highly effective in the induction of labour at term in properly selected cases. In terms of success and failure, dinoprostone vaginal pessary does not differ much from dinoprostone gel.

\section{KEY WORDS}

Labour Induction, Propess, Dinoprostone
Corresponding Author: Dr. Sarmishta $M$,

No 1, Somasundaram Street, T. Nagar, Chennai - 600017, Tamilnadu, India.

E-mail: sarmishta83@yahoo.co.in

DOI: $10.14260 /$ jemds/2021/188

How to Cite This Article:

Mamatha C, Sarmishta M, Dhanalakshmi MG. Efficacy of dinoprostone vaginal pessary for cervical ripening and induction of labour. J Evolution Med Dent Sci 2021;10(12):873-877, DOI: 10.14260/jemds/2021/188

Submission 10-11-2020,

Peer Review 29-01-2021,

Acceptance 05-02-2021,

Published 22-03-2021.

Copyright (C) 2021 Mamatha C. et al. This is an open access article distributed under Creative Commons Attribution License [Attribution 4.0 International (CC BY 4.0)] 


\section{BACKGROUND}

Labour induction is the process which involves artificially initiating the uterine contractions before the onset of true spontaneous labour pains in order to achieve a successful vaginal delivery by medical or surgical means and to avoid operative method of delivery. Induction of labour exceeds 20 $\%$ of all births worldwide, in many countries. ${ }^{1}$ Induction of labour is indicated only when the risk of pregnancy continuation for the mother and the fetus, exceeds the risk associated with induced labour and delivery ${ }^{1}$ The probability of labour induction varies in different women depending on the pregnant women being induced, the indication for induction in all pregnant women and the protocol being used for the women to achieve normal delivery. Different modes of labour induction being used amongst them are prostaglandins E2, prostaglandin E1, syntocinon, Foley's catheter, mifepristone RU 486, artificial rupture of membranes, stripping of membranes but prostaglandins remain a preferred method for cervical ripening and labour induction. ${ }^{1}$ The preparations of prostaglandins PGE2 are available in different forms mainly gel, tablet and pessary (propess) which appear to be equally efficacious in the labour induction (Kelly et al. 2003). Dinoprostone is one of the preferred forms of prostaglandin (PGE 2) which is administered when the cervix has a Bishop's score of $\leq$ six and the rate of vaginal delivery is preferred within 24 hours. $^{2}$ Dinoprostone vaginal pessary offers advantages in the form of single application, ease of administration, less invasiveness, slow release of drug over 24 hours and removal allowing greater dose control, reduction in the number of internal examinations, improved patient compliance.3,4 The pessary has a special retrieval tape that allows it to be removed quickly and easily once there is a problem in induction or when the labour starts. Propess contains a controlled hydrophilic matrix which provides a gradual release of dinoprostone which was introduced in 1995. This preparation is being used for the cervical ripening initiation or for enhancement of cervical ripening in women at term with singleton pregnancy with cephalic presentation. Prostaglandin E2 (PGE2) causes uterine contractions by softening the cervix and dilating the cervix which induces labour. This review summarises the pharmaceutical, pharmacological, pharmacokinetic, and clinical studies conducted with controlled release of prostaglandin E2 and related products. During spontaneous labour, prostaglandin E2 (PGE2) plays a major role in the physiological changes in the delivery of term pregnant women and is an important mediator in uterine contraction and cervical softening and ripening and dilatation of the cervix. It is believed to relax the cervical smooth muscles and also increases the production of glycosaminoglycans within the collagen fibers of the cervix which result in softening and ripening of cervix and dilatation of the cervix leading to delivery. Pregnancy induces the pharmacological action of prostaglandin E2 (PGE2) on the uterus. During pregnancy it causes uterine contractions but in a nongravid uterus it causes uterine smooth muscle relaxation. The uterus is more receptive during term than during pregnancy and the uterine responsiveness depends on the duration of pregnancy. Knowing the physiological actions of endogenous prostaglandin E2 (PGE2), it has been administered as a pharmacological agent to enhance the cervical ripening, softening and dilatation of the cervix thus, inducing labour. Early studies have shown that the extraamniotic administration of prostaglandin E2 led to more convenient intravaginal administration as a gel or pessaries. AIM

\section{METHODS}

This was a prospective observational study conducted at Sri Ramachandra Institute of Higher Education and Research from September 2016 to September 2018. The study included pregnant women with singleton pregnancy and gestational age between 37 weeks -41 weeks of gestation. The study was approved by the ethical committee of Sri Ramachandra Institute of Higher Education and Research, ethics numberCSP-MED / 17 / JUN / 36 / 58. The estimated sample size was 100.

\section{Inclusion Criteria}

- Bishop score $</-6$

- Gestational age $>$ / - 37 weeks

- $\quad$ Singleton pregnancy

- Cephalic presentation

- Parity $<4$

- Hypertensive disorders of pregnancy

- Gestational diabetes mellitus

- Decreased fetal movements

- Oligohydramnios

- Postdatism

- Preterm rupture of membranes

- Intrauterine fetal death

- Intrauterine growth restriction

\author{
Exclusion Criteria for the Study \\ - In active labour \\ - Previous uterine scar \\ - Prior uterine rupture \\ - Prostaglandins hypersensitivity \\ - Malpresentation of foetus \\ - Cephalopelvic disproportion (CPD) \\ - Placenta previa or vasa previa \\ - Suspected abruption \\ - Macrosomia \\ - Genital herpes \\ - Cervical carcinoma \\ - $\quad$ Severe preeclampsia
}

Women who were fulfilling both the inclusion and exclusion criteria were enrolled in the study. Informed consent of all women who underwent the study was obtained. After a detailed history and general examination of the women who were included in the study, an obstetric examination was done. Fetal heart rate was also assessed by Cardiotocogram. All the 100 women were induced with dinoprostone vaginal pessary. All women underwent a sterile per vaginal examination and Bishop's scoring was done. 
- Dinoprostone vaginal pessary stored in freezer between $10^{*} \mathrm{c}$ to $-25^{*} \mathrm{c}$, was removed from freezer just before insertion.

- Single vaginal pessary was administered transversely in the posterior fornix up to 24 hours.

- Patient remained recumbent for 30 minutes after insertion.

- Fetal heart rate and uterine contractions was monitored for 20 minutes every 4 hours.

- After 12 hours and 24 hours Bishop score was reevaluated.

- Data was collected and analysed.

\section{Vaginal Pessary was Removed}

- If its 24 hours from the time of insertion, irrespective of Bishop score and uterine contractions.

- If the patient has entered active phase of labour.

- Artificial rupture of membranes to be performed after 30 minutes followed by oxytocin augmentation up to $32 \mathrm{mu} /$ min if indicated).

- If the patient has adequate uterine contractions (3 contractions lasting for 45 seconds each in 10 minutes).

- If the patient has spontaneous rupture of membranes with favourable cervix (followed by oxytocin augmentation up to $32 \mathrm{mu} / \mathrm{min}$ if indicated).

- In case of uterine tachysystole.

- In case of fetal distress.

- Treatment failure was considered if patient was not in labour even after amniotomy +6 hours of oxytocin with adequate contractions.

- When more than 5 contractions were present in 10 minutes for at least 20 minutes it was diagnosed as uterine tachysystole.

- Uterine hypertonus was diagnosed when a single uterine contraction lasted for at least 2 minutes

- The presence of persistent variable decelerations, persistent late decelerations or bradycardia with absent variability, severe decelerations if present, foetal heart rate abnormality was diagnosed.

\section{Primary Outcome Measures}

$>$ Induction to active phase interval

- $\quad<12$ hours

- $12-24$ hours

- $\quad>24$ hours

$>$ Induction to delivery time

- $\quad<24$ hours

- $\quad>24$ hours

\section{Secondary Outcome Measures}

$>$ Uterine tachysystole

$>$ Need for tocolytic administration

$>$ Need for artificial rupture of membranes + / - oxytocin

$>$ Meconium stained liquor

$>$ Mode of delivery:

- Spontaneous vaginal delivery with episiotomy

- Assisted vaginal delivery (instrumental) with episiotomy

- Caesarean section

Postpartum haemorrhage
$>$ Need for neonatal intensive care unit (NICU) care $>24$ hours

\section{Statistical Analysis}

The statistical analysis mainly comprises the primary outcome, induction delivery time, need for augmentation, mode of delivery, secondary outcomes which have been analysed. The P-value was calculated by chi-square test. The statistical software used was SPSS-16 Statistical Product and Service Solution.

\section{RESULTS}

\begin{tabular}{|cccc|}
\hline Indication for Induction & Primi & Multi & $\begin{array}{c}\text { Total } \\
\text { (Percentage) }\end{array}$ \\
Postdatism & 39 & 12 & $\mathbf{4 2}$ \\
Oligohydramnios & 5 & 4 & $\mathbf{9}$ \\
Decreased fetal movements & 2 & 2 & $\mathbf{4}$ \\
GDM on OHA & 9 & 8 & $\mathbf{1 7}$ \\
GHTN & 3 & 1 & $\mathbf{4}$ \\
PROM & 2 & 1 & $\mathbf{3}$ \\
Non reassuring NST & 2 & 1 & $\mathbf{3}$ \\
GDM on insulin & 3 & 1 & $\mathbf{4}$ \\
ECV & 0 & 1 & $\mathbf{1}$ \\
Overt DM on OHA & 0 & 1 & $\mathbf{1}$ \\
IUGR & 0 & 1 & $\mathbf{1}$ \\
Bad obstetrics history & 1 & 0 & $\mathbf{1}$ \\
Gestational thrombocytopenia & 1 & 0 & $\mathbf{1}$ \\
\hline Total & $\mathbf{6 7}$ & $\mathbf{3 3}$ & $\mathbf{1 0 0}$ \\
\hline Table 1. Indications for Induction of Labour \\
\hline \multicolumn{2}{|l}{}
\end{tabular}

Table 1 shows the common indications for induction. The most common indication was postdatism comprising $42 \%$, followed by $17 \%$ gestational diabetes mellitus (GDM) and $9 \%$ oligohydramnios.

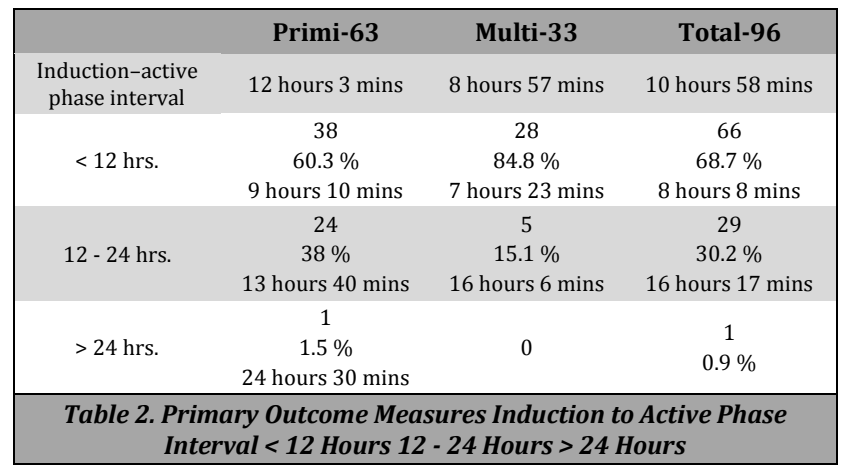

\begin{tabular}{|cccc|}
\hline $\begin{array}{c}\text { Induction- } \\
\text { Delivery Interval }\end{array}$ & $\begin{array}{c}\text { Primi-67 } \\
\text { 17 Hrs. 36 Mins }\end{array}$ & $\begin{array}{c}\text { Multi-33 } \\
\text { 12 Hrs. 19 Mins }\end{array}$ & $\begin{array}{c}\text { Total-100 } \\
\text { 16 Hours 5 Mins }\end{array}$ \\
$<24$ hrs. & 60 & 33 & 93 \\
& $(95.2 \%)$ & $(100 \%)$ & $(93 \%)$ \\
& 14 hours 31 mins & 12 hrs. 19 mins & 15 hrs. 3 mins \\
$>24$ hrs. & 7 & & 7 \\
& $(11.1 \%)$ & 0 & $(7 \%)$ \\
\hline \multicolumn{4}{r}{ Table 3. Induction to Delivery Time $<$ 24 Hours $>$ 24 Hours } \\
\hline
\end{tabular}

Table 2 shows the induction to active phase interval. Out of 100 patients, 4 nulliparous women had failed induction. About $60.3 \%$ of nulliparous women entered active phase of labour within 12 hours with a mean time of 12 hours 3 minutes and $84.8 \%$ of multiparous women entered active phase within 12 hours with a mean time of 8 hours 57 minutes. The average 
time taken for entering active phase of labour was 10 hours 58 minutes. The P-value was 0.13 which is not significant.

Table 3 shows the induction to delivery interval. Out of 100 patients, $93 \%$ of patients delivered within 24 hours. About $95.2 \%$ of nulliparous women delivered within 24 hours with a mean time of 17 hours 36 minutes and $100 \%$ of multiparous women delivered within 24 hours with a mean time of 12 hours 19 minutes. The average induction to delivery time interval was 16 hours 5 minutes. The P-value was 0.124 which is not significant.

\begin{tabular}{|cccc|}
\hline Need for Augmentation & $\begin{array}{c}\text { Primi } \\
\mathbf{6 7}(\mathbf{6 7} \%)\end{array}$ & $\begin{array}{c}\text { Multi } \\
\mathbf{3 3} \mathbf{( 3 3} \boldsymbol{\%})\end{array}$ & $\begin{array}{c}\text { Total } \\
\text { Percent }\end{array}$ \\
\hline Need for arm & 41 & 15 & $\mathbf{5 6 \%}$ \\
Meconium stained liquor (MSL) & 3 & 2 & $\mathbf{5 \%}$ \\
Grade 1 MSL & 0 & 1 & $\mathbf{1 \%}$ \\
Grade 2 MSL & 1 & 1 & $\mathbf{2} \%$ \\
Grade 3 MSL & 2 & 0 & $\mathbf{2 \%}$ \\
Need for oxytocin augmentation & 35 & 8 & $\mathbf{4 3 \%}$ \\
\hline Failed induction & 4 & 0 & $\mathbf{4 \%}$ \\
\hline \multicolumn{2}{c}{ Table 4. Need for Augmentation } \\
\hline
\end{tabular}

Table 4 shows the overall augmentation of labour with artificial rupture of membranes and oxytocin. $56 \%$ of patients underwent augmentation of labour with artificial rupture of membrane (ARM) and $43 \%$ of patients required oxytocin augmentation. Only $8 \%$ of multiparous women required oxytocin augmentation. Meconium-stained liquor was noted in $5 \%$ of cases. Failed induction was noted in 4 nulliparous women. The P-value was 0.02 which is not significant.

\begin{tabular}{|c|c|c|c|c|}
\hline Mode of Delivery & Primi-67 & Multi-33 & $\begin{array}{l}\text { Total- } \\
100\end{array}$ & P-Value \\
\hline $\begin{array}{c}\text { Spontaneous vaginal delivery with } \\
\text { episiotomy }\end{array}$ & $49(73.1 \%)$ & $31(93.9 \%)$ & $80 \%$ & 0.11 \\
\hline $\begin{array}{c}\text { Assisted vaginal delivery with } \\
\text { episiotomy }\end{array}$ & $4(5.9 \%)$ & $1(3 \%)$ & $5 \%$ & 0.11 \\
\hline Caesarean section & $14(20.8 \%)$ & $1(3 \%)$ & $15 \%$ & 0.014 \\
\hline Caesarean section indication & Total-14 & Total-1 & $\begin{array}{c}\text { Total-15 } \\
\%\end{array}$ & \\
\hline Pathological cardiotocography (CTG) & 3 & 0 & $20 \%$ & \\
\hline Foetal distress & 6 & 1 & $46.6 \%$ & \\
\hline Arrest of descent & 1 & 0 & $13 \%$ & \\
\hline Failed induction & 4 & 0 & $4 \%$ & \\
\hline \multicolumn{5}{|c|}{ Table 5. Mode of Delivery and Statistical Analysis } \\
\hline
\end{tabular}

As shown in Table 5, $80 \%$ of the patients delivered by spontaneous vaginal delivery with episiotomy, $5 \%$ of patients delivered by assisted vaginal delivery (vacuum) and $15 \%$ of patients delivered by lower segment Caesarean section. The most common indication for Caesarean section being fetal distress comprising of about $46.6 \%$. Almost $97 \%$ of multiparous women delivered vaginally. The P-value of normal delivery and vacuum delivery was 0.011 which is highly significant. The P-value of Caesarean section was 0.014 which is also highly significant.

\begin{tabular}{|cc|}
\hline Uterine Tachysystole & $\mathbf{4} \%$ \\
PPH & $1 \%$ \\
Others: nausea, vomiting, pyrexia, diarrhoea, headache & $\mathrm{Nil}$ \\
Baby weight $<2.5 \mathrm{kgs}$ & $5 \%$ \\
Baby weight $>2.5 \mathrm{kgs}$ & $95 \%$ \\
Apgar less than or equal to 7 at 1 minute & $6 \%$ \\
Apgar less than or equal to 7 at 5 minutes & $1 \%$ \\
\hline NICU admissions ( $>24$ hrs.) & $1 \%$ \\
\hline Table 6. Secondary Outcomes & \\
\hline
\end{tabular}

As shown in Table 6, $6 \%$ of babies had Apgar $</-7$ at 1 minute and $1 \%$ of babies had Apgar $</-7$ at 5 minutes. Only $1 \%$ of the babies required NICU care $>24$ hours. No adverse maternal and neonatal outcome was noted in the present study. $95 \%$ of babies weighed more than $2.5 \mathrm{~kg}$. Mild atonic postpartum haemorrhage was noted in $1 \%$ of the cases which was medically managed. Uterine tachysystole was noted in 4 $\%$ of cases where only 1 patient required tocolytic administration, 2 patients delivered vaginally, and the other 2 patients delivered by Caesarean section. No allergic reactions to the drug were noted. There were no neonatal deaths reported. The P-value was 0.04 which is not significant.

\section{DISCUSSION}

Table 1 shows the common indications for induction. The most common indication was postdatism comprising $42 \%$, followed by $17 \%$ GDM and $9 \%$ oligohydramnios. As shown in Table 2, in the present study, $60.3 \%$ of nulliparous women entered active phase within 12 hours with a mean time of 12 hours 3 minutes and $84.8 \%$ of multiparous women entered active phase within 12 hours with a mean time of 8 hours 57 minutes. The mean induction to active phase interval being 10 hours 58 minutes which is comparable with the study by Marconi et al. ${ }^{3}$ Also, the time interval from induction to the active phase is shorter in this study when compared to other studies.

As shown in Table 2, the mean induction to delivery time interval in the present study was 16 hours 5 minutes. Of which $93 \%$ patients delivered within 24 hours. In total, $95.2 \%$ nulliparas delivered within 24 hours and $100 \%$ of multiparas delivered within 24 hours. The above results were comparable with the study by Macroni et al. ${ }^{3}$ Also, the time interval from induction to delivery is shorter in this study when compared to other studies.

As shown in Table $4,43 \%$ of patients (35\% of nulliparous and $8 \%$ of multiparous) of the present study required augmentation with oxytocin which is comparable with study by Macroni et al. ${ }^{4}$ Conversely, only $15 \%$ of patients required augmentation of labour in the study by Pitale et al. ${ }^{5}$

As depicted in Table 5, $80 \%$ delivered by spontaneous vaginal delivery, $5 \%$ delivered by assisted vaginal delivery (vacuum), $15 \%$ delivered by lower segment Caesarean section. The commonest indication for Caesarean section being fetal distress comprising $46.6 \% .97 \%$ of multiparous women delivered vaginally. Similar results were obtained in the study by Marconi et al. ${ }^{4}$ However, rates of normal delivery were high when compared to other studies by Zhao et al. Kalkat et al.6,7 Conversely, the present study has a low Caesarean section rate in multiparous (3\%), regardless of the Bishops score. The total Caesarean section rate was $15 \%$ in these patients which is comparable with rates of spontaneous labour at our institution (13.7\%). The large use of amniotomy / syntocinon is the one of the possible explanation. ${ }^{8}$ Out of the total, $43 \%$ of our patients' received additional augmentation which might have caused an increase in the number of vaginal delivery where patients delivered successfully and further was decrease in the number of Caesarean section. Apgar $</-7$ at 1 minute and 5 minutes is comparable with Macroni et al. 
study. However, there were no NICU admissions more than 24 hours in other studies by Pitale et al. and Kalkat et al.5,7

As shown in Table 6, uterine tachysystole was observed in four patients. In these cases, under strict aseptic precautions vaginal pessary was removed immediately and the cervical findings were favourable in all the 4 cases. Only $1 \%$ of patients had postpartum haemorrhage (PPH) and none of the patient had drug allergic reaction in the present study. The present study confirms that the use of dinoprostone vaginal pessary is safe and does not cause uterine tachysystole and decreases neonatal and maternal morbidity and mortality for up to 24 hours. Vaginal pessary used in primigravida with an unfavourable cervix had a decreased rate of post-partum blood loss less than $500 \mathrm{ml}$ when compared to dinoprostone gel treated women in other studies. In conclusion, the present study shows that dinoprostone vaginal pessary are efficient in achieving cervical ripening, softening and cervical dilatation and successful vaginal delivery both in primiparous and multiparous with negligible maternal and fetal / neonatal side effects. ${ }^{9}$ The PGE2 vaginal pessary seems more convenient in primigravida patients, especially when the pre induction Bishop score is less than 4, given its handiness, one administration, possible application overnight, easy removal in case of side effects, less vaginal examinations and most important is the decreased risk of postpartum haemorrhage. ${ }^{10}$

\section{Summary}

The present study involves 100 antenatal patients undergoing labour induction with dinoprostone vaginal pessary, among whom $67 \%$ were nulliparous women and $33 \%$ were multiparous women. About $46 \%$ of patients were under the age group of 25 to 28 years with the most common medical disorder complicating pregnancy which is gestational diabetes. Nearly $43 \%$ of patients were induced around 40 weeks of gestation with the mean Bishop score of 4 and the commonest indication of induction being postdatism. The average induction to active phase interval was 10 hours 58 minutes. About $60.3 \%$ of primigravida entered active phase within 12 hours with a mean induction to active phase time interval of 12 hours 03 minutes and $95.2 \%$ of primigravida delivered $<24$ hours with a mean induction to delivery time interval of 17 hours 36 minutes. $4 \%$ of primigravida had failed induction. About $84.8 \%$ of multigravida entered active phase within 12 hours with mean induction to active phase time interval of 08 hours 57 minutes and $100 \%$ of multigravida delivered $<24$ hours with a mean induction to delivery time interval of 12 hours 19 minutes. $43 \%$ of patients required augmentation of labour with oxytocin. Out of 100 patients, 80 $\%$ of patients delivered by spontaneous vaginal delivery with episiotomy, $5 \%$ of patients delivered by assisted vaginal delivery with episiotomy and $15 \%$ of patients delivered by Caesarean section. Almost $97 \%$ of multiparous women delivered vaginally. Uterine tachysystole was noted in $4 \%$ of cases. Mild atonic postpartum haemorrhage was noted in 1 patient who was medically managed. Only $1 \%$ of babies required NICU care more than 24 hours. No adverse maternal and neonatal outcome was noted in the present study.

\section{CONCLUSIONS}

Dinoprostone vaginal pessary is highly effective in the induction of labour at term in properly selected cases. In terms of success and failure, dinoprostone vaginal pessary does not differ much from dinoprostone gel.

Data sharing statement provided by the authors is available with the full text of this article at jemds.com.

Financial or other competing interests: None.

Disclosure forms provided by the authors are available with the full text of this article at jemds.com.

\section{REFERENCES}

[1] WHO recommendations for induction of labour. World Health Organization: Geneva, 2011.

[2] ACOG Practice Bulletin No. 107: Induction of labor. Obstet Gynecol 2009;114(2 Pt 1):386-97.

[3] National Institute for Health and Care Excellence Induction of labour. (Clinical guideline 70). NICE 2008. www.nice.org.uk/guidance/cg70

[4] Marconi AM, Bozzetti P, Morabito A, et al. Comparing two dinoprostone agents for cervical ripening and induction of labor: a randomized trial. Eur J Obstetr Gynecol Reprod Biol 2008;138(2):135-40.

[5] Pitale DL. Effectiveness of dinoprostone vaginal pessary in induction of labour at term. Int J Reprod Contracept Obstet Gynecol 2017;6(12):5528-31.

[6] Zhao L, Lin Y, Jiang TT, et al. Vaginal delivery among women who underwent labor induction with vaginal dinoprostone (PGE2) insert: a retrospective study of 1656 women in China. The Journal of Maternal-Fetal \& Neonatal Medicine 2017;32(10):1721-7.

[7] Kalkat RK, McMillan E, Cooper H, et al. Comparison of Dinoprostone slow release pessary (Propess) with gel (Prostin) for induction of labour at term-a randomised trial. J Obstetr Gynaecol 2008;28(7):695-9.

[8] Mizrachi Y, Levy M, Bar J, et al. Induction of labor in nulliparous women with unfavorable cervix: a comparison of foley catheter and vaginal prostaglandin E2. Arch Gynecol Obstet 2016;294(4):725-30.

[9] Hiersch L, Borovich A, Gabbay-Benziv R, et al. Can we predict successful cervical ripening with prostaglandin E2 vaginal inserts? Arch Gynecol Obstet 2016;295(2):343-9.

[10] Jozwiak M, Bloemenkamp KWM, Kelly AJ, et al. Mechanical methods for induction of labour. Cochrane Database Syst Rev 2012;3:CD001233. 